\title{
La buena educación: nociones del alumnado de un bachillerato universitario acerca de la calidad educativa
}

Norma Graciella Heredia Soberanis y Rubi Surema Peniche Cetzal

\section{Introducción}

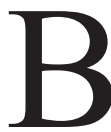
ajo la ideología neoliberal que domina actualmente el campo de la educación formal, el término "calidad" está presente en la redacción de metas y objetivos estratégicos incluidos en los planes institucionales, por lo que técnicamente representa un macrocriterio para evaluarla. Para Gago (2002), usar sólo el término "calidad", sin ningún calificativo que lo acompañe, es ambiguo, ya que mencionar que algo es de calidad es una obviedad, pues todo tiene calidad, aunque sea mala. Este autor señala que lo fundamental de la evaluación radica en el adjetivo que ha de agregarse al sustantivo "calidad", por lo que encontrar el adjetivo más adecuado, es decir, la calificación correcta y verdadera de la calidad de algo, es el propósito de la evaluación; de tal forma que al hablar de la calidad de los programas educativos, lo deseable es contar con procesos evaluativos transparentes y precisos en sus paradigmas, enfoques y métodos, para que los resultados que revelen sean creíbles, válidos y confiables.

Atendiendo a las necesidades por determinar un camino hacia una educación de calidad, en México, la definición del concepto fue operacionalizada con las siguientes dimensiones: relevancia, eficiencia, pertinencia, impacto, equidad, eficacia interna y eficacia externa (Instituto Nacional para la Evaluación de la Educación [INEE], 2011). Las dimensiones de eficacia y eficiencia muestran elementos traducidos en estándares cuantitativos calculados con base en porcentajes, tasas o índices, como: de retención, deserción, promoción, eficiencia terminal, entre otros; sin embargo, es interesante que en la definición del constructo calidad, contemporáneamente sea considerada la equidad.

Entonces, ¿lo educativo sería de calidad haciendo referencia al componente distributivo en cuanto al acceso, permanencia y conclusión de la escolarización mínima obligatoria, aunado a que la educación proporcionada sea igualmente justa respecto a la igual utilidad de lo aprendido para la diversidad de características y puntos de partida de cada persona?

Aunque sea muy aventurado, se puede decir que el término calidad se encuentra muy desgastado en los discursos políticos y educativos, y hasta ahora, a pesar de los esfuerzos a nivel mundial, nacional y local, consistentes en la implementación de reformas para los diferentes niveles de los sistemas educativos, la educación formal ha sido insuficiente para que todo ciudadano pueda ejercer el derecho a ser y estar educado.

Actualmente, la educación media superior (EMS) en México enfrenta retos de gran consideración, entre ellos se encuentra la baja cobertura, problemas de equidad, tasas altas de abandono escolar, profesores y directivos con debilidades en su formación, bajos resultados educativos, calidad diferenciada en los servicios, falta de oportunidad para continuar con estudios de nivel superior, deficiencias en la infraestructura y el equipamiento 
de las escuelas, desarticulación con la demanda laboral, así como alta presencia de violencia y conducta de riesgo entre los estudiantes (Ortega, 2014). Para dar respuesta a estas problemáticas, de manera local la Universidad Autónoma de Yucatán (UADY) elaboró en el año 2012 una reestructuración de su primer modelo educativo, al cual denominó Modelo Educativo para la Formación Integral (MEFI), basado en ofertar una educación de alta calidad para todos los niveles educativos que imparte, entre ellos, los bachilleratos generales, siendo para la península de Yucatán la oferta educativa prioritaria para cursar EMs.

¿Qué significa una educación de calidad para el alumnado de EMs? A continuación, se describen los resultados encontrados mediante la realización de tres grupos focales y cuatro entrevistas individuales a estudiantes y egresados de un bachillerato universitario con énfasis en la interacción comunitaria, ofertado por la UADY. En total participaron 36 estudiantes (12 por cada grupo focal: seis hombres y seis mujeres), los dos mejores promedios de la primera cohorte egresada del bachillerato, dos estudiantes (un hombre y una mujer) con el más bajo rendimiento académico por su promedio inferior a 80 puntos, así como dos estudiantes (un hombre y una mujer) en situación de irregulares por haber reprobado más de tres asignaturas.

Las nociones de calidad expresadas por el alumnado, con base en Pérez (2004: 19-22), representan ideas complementarias de: a) percepción del servicio como satisfactorio, criterio básico de la calidad total, uno de los enfoques de movimientos de la calidad; y b) aquello que afecta la naturaleza de las cosas, enfoque absoluto bajo el cual la calidad equivale a una cualidad, clase, categoría, entendida como sinónimo de bueno, excelente o elevado. Para el alumnado, una educación de calidad, en sus propias palabras, es "una buena educación".

En la Figura 1 puede observarse la representación de los factores identificados con base en las opiniones del alumnado participante.

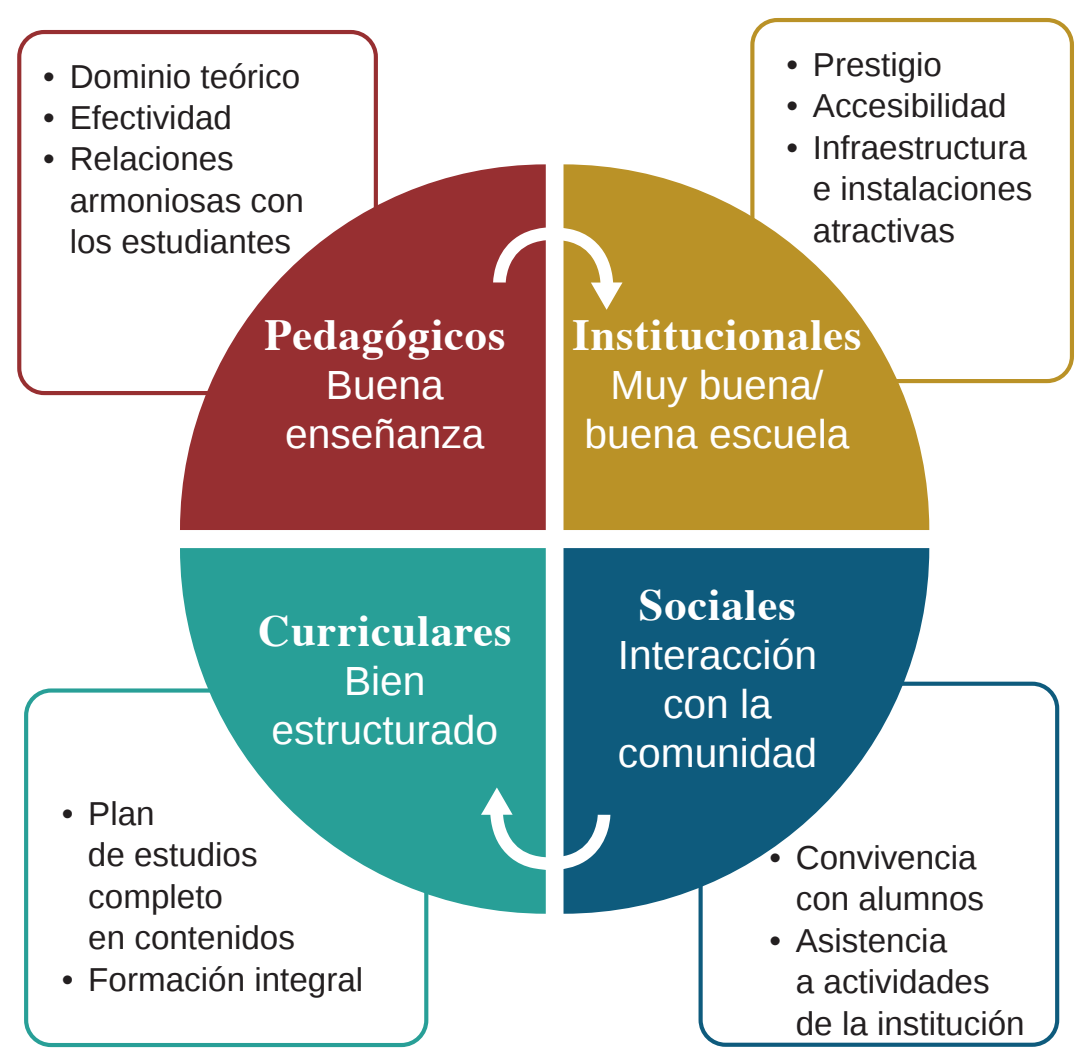

Figura 1. Factores asociados a nociones de una educación de calidad. 


\section{Factores institucionales}

Como factores institucionales los alumnos mencionaron elementos de organización escolar, ambiente social y relaciones externas. Para ellos, el ser una muy buena escuela implica que la gente conocida hable bien de ella y que la recomiende; es decir, involucra el reconocimiento social y la posesión de prestigio en el medio. Según Poulsen (2006), el prestigio es relacional y se basa en el reconocimiento y la evaluación que un público hace de un autor. Puede ser considerado como un atributo personal y sólo puede adjudicarse si un público o fuente lo acepta; en ese sentido, en términos del prestigio, no importa lo que eres, sino lo que la gente piensa que eres. Otro componente fue la accesibilidad que ésta brinda para ingresar y permanecer en ella; implica, por una parte, cobrar cuotas de inscripción o pagos económicos en contraste con otras instituciones educativas similares, y por otra, tener una ubicación cercana al domicilio de los estudiantes o facilidad de transporte.

\section{Factores pedagógicos}

La noción de buena enseñanza estuvo ligada a una intervención del profesorado que logre un cambio percibido en el alumnado respecto a un mejor dominio teórico-práctico de las disciplinas preponderantes en el currículo, como son comunicación y matemáticas; claro, en contraste con el estado de conocimiento previo auto-identificado por el alumno. Centrarse en los buenos resultados educativos, desde el punto de vista de Martínez (2008: 35), pone "el énfasis en el mundo real, exterior, en lo observable, y no en los procesos subjetivos del que reproduce la competencia".

Otro aspecto que configura las nociones de una buena enseñanza es la exigencia en el alumnado de un nivel de desempeño elevado, así como fomentar en éste la dedicación hacia el estudio y la competitividad; sin embargo, a pesar de que una parte del alumnado tiene la idea de que la buena enseñanza implica exigencia, otros estudiantes piensan que es necesario un límite en ella. La noción de buena enseñanza también estuvo basada en la posesión de conocimientos en gran cantidad por parte del profesorado. Y aunque en la literatura de evaluación del desempeño docente ha sido ampliamente debatido
« Al hablar de la calidad de los

programas educativos, lo deseable

es contar con procesos evaluativos

transparentes y precisos en sus

paradigmas, enfoques y métodos para

que los resultados que revelen sean

creíbles, válidos y confiables. \

qué tanto un alumno puede evaluar el dominio teórico del profesorado, lo que se hizo evidente es que la idea de tener muchos conocimientos forma parte de "ser un buen profesor".

Con base en las ocho competencias que definen el Perfil del Docente del Sistema Nacional de Bachillerato: 1. Organiza su formación continua a lo largo de su trayectoria profesional; 2. Domina y estructura los saberes para facilitar experiencias de aprendizaje significativo; 3. Planifica los procesos de enseñanza y de aprendizaje atendiendo al enfoque por competencias, y los ubica en contextos disciplinares, curriculares y sociales amplios; 4. Lleva a la práctica procesos de enseñanza y de aprendizaje de manera efectiva, creativa e innovadora a su contexto institucional; 5 . Evalúa los procesos de enseñanza y de aprendizaje con un enfoque formativo; 6 . Construye ambientes para el aprendizaje autónomo y colaborativo; 7. Contribuye a la generación de un ambiente que facilite el desarrollo sano e integral de los estudiantes; y 8. Participa en los proyectos de mejora continua de su escuela y apoya la gestión institucional (Secretaría de Educación Pública [SEP], 2008, acuerdo número 447); cruzando los factores pedagógicos mencionados por los estudiantes entrevistados, se pudo observar que las competencias 2, 3 y 4 son fundamentales desde la experiencia del alumnado.

También se comentó en los grupos focales la idea de que un buen maestro es una buena persona. Según lo dicho por los estudiantes, más allá de la tarea de enseñar contenidos, algunos profesores de bachillerato han logrado establecer relaciones armoniosas, cordiales, de respeto, empatía y solidaridad con ellos, llegando incluso a ser percibidas por un alumno como una relación de amistad. El ser tomado en cuenta como persona es una idea clave que representa la ruptura de esquemas tradicionales respecto al rol que desempeña el profesorado, entonces surge la idea de un profesorado exigente y a la vez 


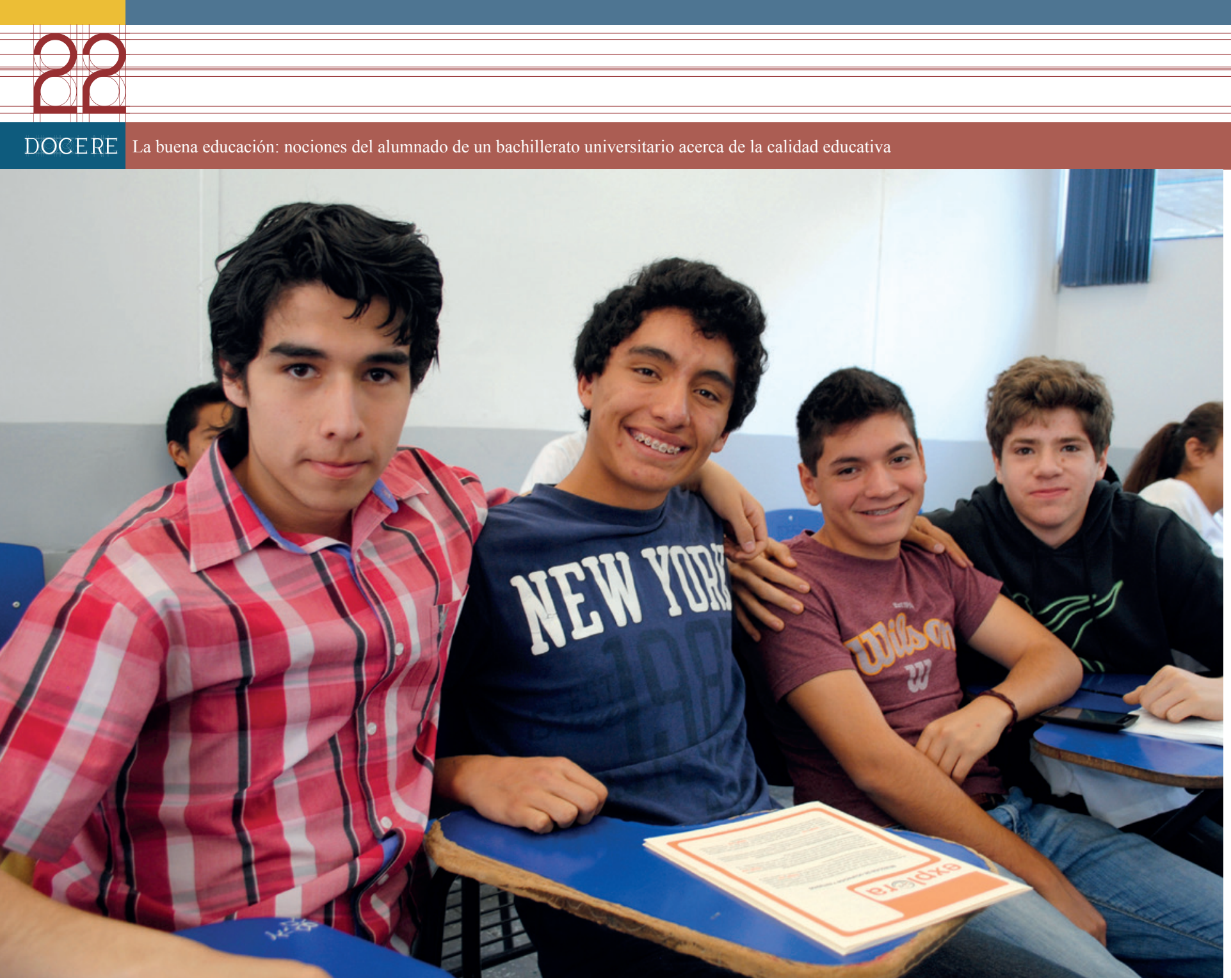

humano. Esa humanidad hacia ellos, por parte del profesorado, permite establecer un vínculo afectivo y, según López (1985:10), establecer este vínculo es una necesidad tan primaria como comer, por lo que esos enlaces, una vez establecidos, "constituyen una poderosa fuerza de unión con los demás y uno de los motivos más fuertes de conducta prosocial". La creación del vínculo afectivo le posibilita al profesor influir en el estudiante, por lo que cumplir sus exigencias académicas y seguir sus recomendaciones se acepta y naturaliza como el rol que le corresponde desempeñar en la escuela, aunque sea pedagógicamente discutible.

\section{Factores curriculares y sociales}

Respecto al plan de estudios, se hizo notoria la idea de que una buena educación implica un contenido curricular extenso en cuanto a la presencia de un mayor número de asignaturas, comparado con otros planes de estudio de bachillerato; y no sólo extenso, sino también variado, al permitirle al alumnado la posibilidad de tener una formación en diferentes áreas disciplinares ligadas a las carreras profesionales, concepto básico en la literatura del campo curricular para hacer referencia a una noción de la flexibilidad curricular.

Otra idea respecto al currículo que representa una buena educación está en el aprendizaje de actitudes positivas para establecer relaciones sociales; se hizo referencia al desarrollo de actitudes y valores sociales; se tiene la percepción de haber aprendido a ser más sociable, así como la convivencia, la generosidad, el ser solidario, el dialogar con la familia y llevarse mejor con compañeros, amigos y su comunidad. En otras palabras, han integrado a sus nociones de una buena educación el que ésta abarque la formación integral, una formación que toca su vida personal, social y familiar, más allá del aprendizaje de las disciplinas. 
La apertura de la escuela, al permitir la participación de investigadores y miembros de grupos sociales que trabajan de forma extraescolar con los estudiantes en actividades del cuidado de la salud, uso de redes sociales, fomento a la lectura, entre otras, también ha ocasionado cambios en la percepción de los estudiantes acerca de lo que es una buena escuela, centrándose en que ésta incluye actividades de interés para la salud y el ocio, además de permitir con naturalidad el ser sujetos de investigación para conocer sus características somatológicas, físicas y psicológicas.

En el texto anterior puede observarse la idea de que una buena escuela incluye la presencia externa de profesionistas preocupados por el bienestar de los estudiantes. Se considera como natural que las autoridades educativas, investigadores y personal de instituciones externas centren su interés en ellos para ayudarlos a salir adelante.

Otra noción de buena educación está ligada a la percepción de que ésta logre cambios positivos en la vida de los estudiantes, ya que, según experiencias personales, debe propiciar formas de pensar y sentir asociadas con la madurez psicosocial esperada en la vida adulta. En este caso, una buena educación tiene como característica transformar al joven en adulto, incorporando en el alumnado deseos de superación, así como la manifestación de formas de pensar que representen madurez, como estar consciente de los problemas sociales, el ser responsable en la escuela en cuanto a estudiar y hacer tareas, así como ser respetuoso con las autoridades, sus familias y solidario con su comunidad.

\section{Conclusión}

Se manifiesta, a través de las palabras expresadas por el alumnado entrevistado, la expectativa y el derecho como ciudadanos de recibir una buena educación, encontrando una contradicción, transitando entre la justicia y la injusticia, ya que, por una parte, asumen que el ser justos con su formación implica otorgarles, como parte de un grupo social selecto, una educación desigual e injusta, es decir, una educación de élite que otros grupos sociales no reciben, siendo cómplices de una injusticia social; sin embargo, dado las características que implica esta educación de élite, ellos perciben igualmente como necesario el vivir un rol de alumno que resulta injusto para los intereses sociales de una vida juvenil posmoderna, lo cual ya no importa, porque eso es lo justo para poder sobrevivir a la vida universitaria neoliberal.

\section{Fuentes de consulta}

Gago, A. (2002). Apuntes acerca de la Evaluación Educativa. México: Secretaría de Educación Pública.

Instituto Nacional para la Evaluación de la Educación (INEE). (2011). La educación media superior en México. Informe anual 20102011. México: INEE.

López, F. (1985). La formación de los vínculos sociales. España: Servicio de Publicaciones del Ministerio de Educación y Ciencia.

Martínez, J. B. (2008). Globalización de los discursos de calidad y evaluación y su impacto en la identidad del profesorado. En J. B. Martínez Rodríguez y J. L. Aróstegui. Globalización, posmodernidad y educación. La calidad como coartada neoliberal. España: Universidad Internacional de Andalucía/ Akal.

Ortega, S. (2014). Retos y desafíos de la EMs en el marco de la reforma. Conferencia Magistral, Aguascalientes.

Pérez, A. (2004). La cultura escolar en la sociedad neoliberal. ( $4^{\mathrm{a}}$ ed.) España: Morata.

Poulsen, C. (2006). El prestigio como factor discriminatorio en la universidad. En E. Pérez et al. (Coords.). Ciencia, tecnología y género en Iberoamérica. España: Ministerio de Educación y Ciencia-csic-Consejo Superior de Investigaciones Científicas.

Secretaría de Educación Pública (SEP). (29 de octubre de 2008). Acuerdo número 447 por el que se establecen las competencias docentes para quienes impartan educación media superior en la modalidad escolarizada. Disponible en: http://dof.gob.mx/nota_detalle. php? codigo $=5066425 \&$ fecha $=29 / 10 / 2008$.

Universidad Autónoma de Yucatán. (2012). Modelo Educativo para la Formación Integral. Yucatán, México: UADY. 